\title{
MECHANICAL ANALYSIS FOR THE DESIGN \\ OF A FIBRE REINFORCED PELVIC PROSTHESIS WITH THE AID OF CAD/CAE-TECHNIQUES
}

\author{
M.R.S. Huisman, M. Maier and S. Kerth \\ Institut far Verbundwerkstoffe GmbH \\ University of Kaiserslautern, Germany
}

\begin{abstract}
Analysis of the mechanical behaviour of a human pelvis is done with and without implanted prosthesis. Results of the analyses are used as boundary conditions in the process of the design of a carbon fibre reinforced pelvic prosthesis. In order to include the analysis part in the whole prosthesis design process, the transfer from the image analysis part to a 3D finite element model is discussed with respect to the interfaces to other systems.
\end{abstract}

\section{INTRODUCTION}

With the resection of malignant tumours in the human pelvis, a demand for well-designed pelvic prostheses is seen. The requirements for a new kind of custom-made prosthesis involve, e.g. new materials and data transfer from image processing, like computer tomography (CT) or Magnetic Resonance Imaging (MRI), to CAD/CAE-models. A flow chart of the whole process cycle from determination of a tumour to the implantation of a prosthesis can be seen in Figure 1.

A precondition for automizing the whole process cycle is to investigate each part and the interfaces between the different parts. In this study the generation of a 3D finite element model of a real human pelvis is demonstrated. Here the data transfer between $C T$ and $\mathrm{CAD} / \mathrm{CAE}$ systems is taken into account.

The analysis of the pelvis is performed for the cases with and without prosthesis by simulation of standing and quasi-static walking. The elastic properties of the prosthesis are varied to obtain knowledge about the influence of the material type on the mechanical behaviour of the pelvic bone.

\section{DATA TRANSFER FROM COMPUTER TOMOGRAPHY TO A FINITE ELEMENT MODEL}

The first step is the translation of CT scans into readable data for the $\mathrm{CAD}$ system. These $\mathrm{CT}$ scans are in the transverse plane of the human body and have a typical distance of two millimeters. A programme which distinguishes between different grey values of the CT scans, plots contour lines of the bone. This results in a wire frame model built up of layers of $2 \mathrm{D}$ contour lines

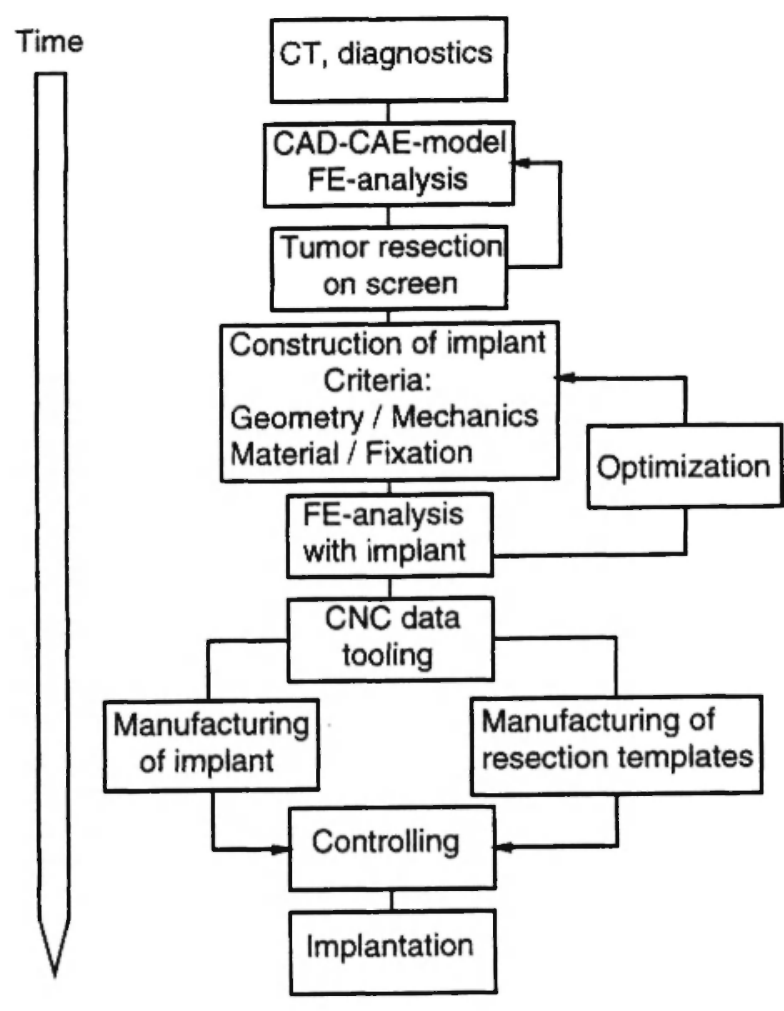

Fig. 1: Flow chart of the design process of a pelvic prosthesis. 


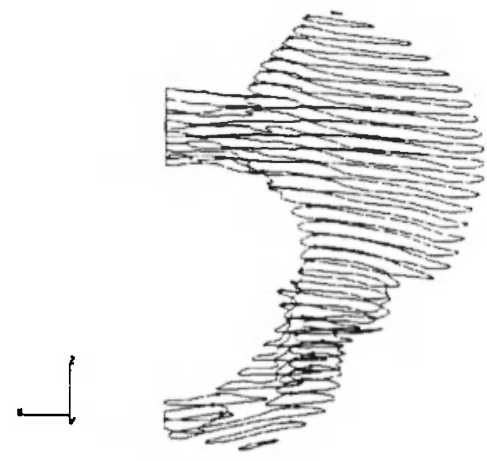

(a)

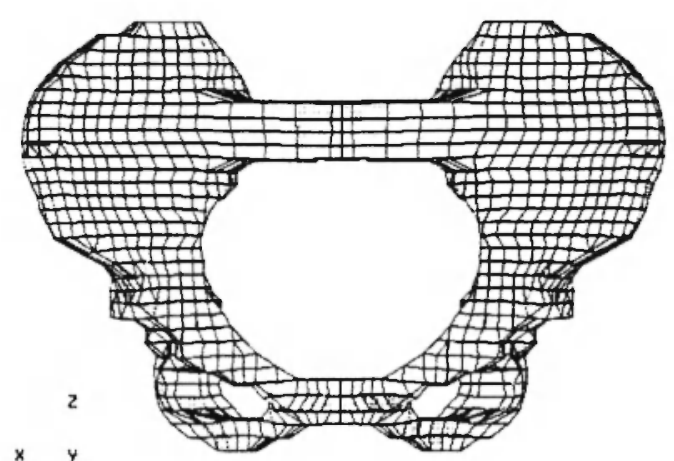

(b)

Fig. 2: Wire frame of CT scans and the 3D finite element mesh of the pelvis.

(Figure 2a). These data are formatted in a way (VDA-FS point set) that they can be used as a basis for the CAE-model and as a basis for a $\mathrm{CNC}$ dataset to mill a foam model of the pelvis.

The second step is the generation of a $3 D$ CAD model from the wire frame. Due to the very complex geometry of the pelvis, difficulties occur when the mesh is generated automatically with standard software. This results in badly shaped elements due to small edge angles and twisting. Therefore, the CAD structure is subdivided into smaller parts with simple cubic shapes. These parts can be meshed automatically with 8-noded solid elements with good results concerning the element shape /1/.

For parts of the structure that still cannot be adequately meshed with 8-noded solid elements, 6-noded wedge elements were created. The resulting $3 \mathrm{D}$ mesh can be seen in Figure 2b. To minimise the amount of work in the investigation of this part of the design process, just one pelvic half is modelled. This part is mirrored after meshing to represent the other half.

\section{SIMULATION OF THE MECHANICAL BEHAVIOUR OF A PELVIS}

The 3D finite element model distinguishes between two bone types, cortical bone and spongy bone. The cortical bone is modelled with the outer layer of solid elements, while the spongy bone is modelled with the inner part of the mesh (Figure 3). Furthermore, the pubic symphysis, the joint between the two pelvic halves, is modelled by a low modulus cartilage. The sacrum, being the lowest part of the trunk, is modelled with solid elements having the elastic properties of cortical bone. The lower part of the sacrum is not

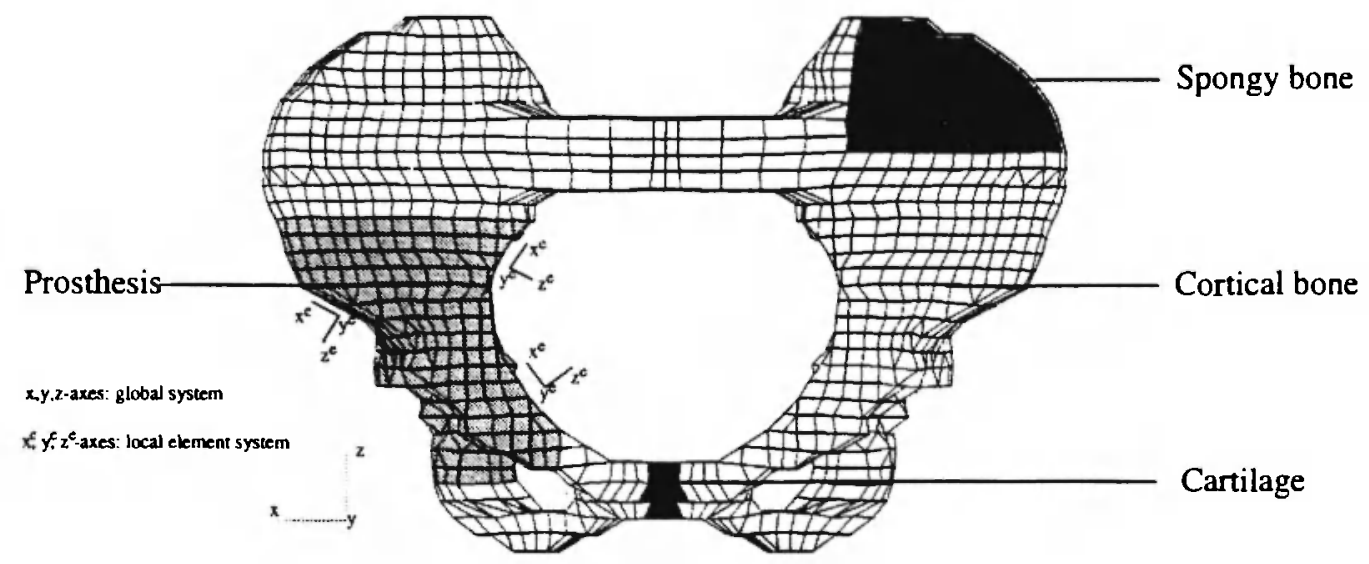

Fig. 3: Location of material regions of the pelvic model. 
modelled to reduce simulation time. Table 1 shows the variety of the mechanical properties of the bone. For the analysis a mean value of the range of Young's moduli is chosen.

The geometry of the implant is chosen to be the same as the already existing mesh of the bone. This enables the comparison of the mechanical responses of the pelvis on changes in the type of material of the implant (Table 2).
Boundary conditions for standing on two legs result from simple biomechanical considerations, while the boundary conditions and muscle forces during walking are based on analyses of Seireg and Arvikar $/ 2 /$. The simulation of walking is confined to quasi-static walking at $15 \%$ of the gait cycle (heel strike). This is the moment of maximum load.

The hip joint reaction forces work at the acetabular surface (Figure 4). The proximal side of the sacrum,

Table 1

Mechanical properties of bone, Keaveny /3/, Dalstra /4/.

\begin{tabular}{|l|l|l|l|}
\hline Material & Range of E-modulus & Chosen E-modulus & Poisson's ratio \\
\hline Cortical bone & $8000-24000 \mathrm{MPa}$ & $17000 \mathrm{MPa}$ & $0.3[1]$ \\
\hline Spongy bone & $200-1500 \mathrm{MPa}$ & $1000 \mathrm{MPa}$ & $0.2[1]$ \\
\hline Cartilage & $1-14 \mathrm{MPa}$ & $14 \mathrm{MPa}$ & $0.48[1]$ \\
\hline
\end{tabular}

Table 2

Material properties of fibre reinforced polymers of MAN Ceramics Ltd., Deggendorf, Germany /5/

\begin{tabular}{|l|l|l|l|l|}
\hline \multirow{2}{*}{$\begin{array}{l}\text { Polymer } \\
\text { Reinforcement }\end{array}$} & \multicolumn{2}{|l|}{ Young's moduli } & $\begin{array}{l}\text { Poisson's } \\
\text { ratio }\end{array}$ & $\begin{array}{l}\text { Shear } \\
\text { modulus }\end{array}$ \\
\cline { 2 - 5 } & El & E2 & nl2 & G12 \\
\hline Short Fiber & $8000[\mathrm{MPa}]$ & $8000[\mathrm{MPa}]$ & $0.37[1]$ & $2900[\mathrm{MPa}]$ \\
\hline Endless Fiber & $170000[\mathrm{MPa}]$ & $8800[\mathrm{MPa}]$ & $0.26[1]$ & $5500[\mathrm{MPa}]$ \\
\hline Woven Fabric & $45000[\mathrm{MPa}]$ & $45000[\mathrm{MPa}]$ & $0.04[1]$ & $3100[\mathrm{MPa}]$ \\
\hline
\end{tabular}

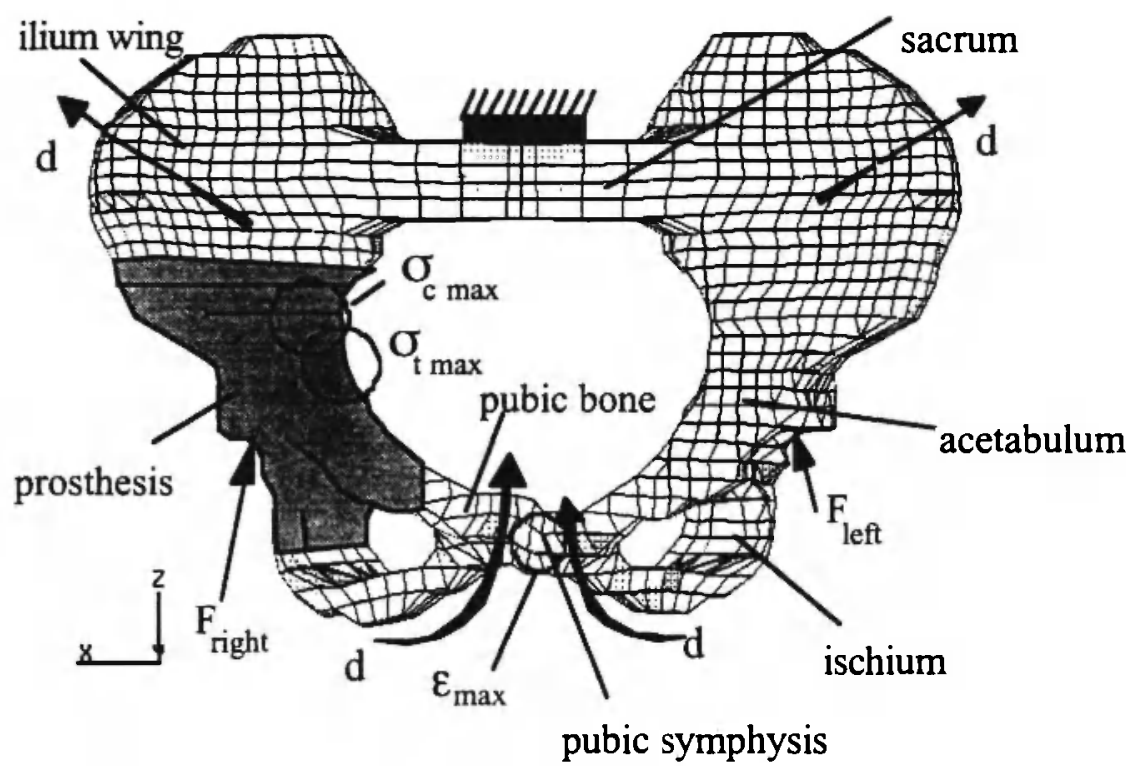

Fig. 4: Deformation of the pelvis at $15 \%$ of the gait cycle. 
representing the connection to the spine, is used as fixation.

\section{RESULTS}

Generally the deformation and stresses during walking are much higher than in the case of standing. Therefore, only results of the simulation of walking at $15 \%$ of the gait cycle, heel strike of the right foot, are shown in Figure 4 and Figure 5.

It has been found that implanting a prosthesis results in a globally stiffer behaviour than in the case of a healthy pelvis. This can be clearly demonstrated by the reduced strains of the cartilage in the pubic symphysis (Figure 5). A change in the Young's modulus of the prosthesis has no significant influence on the strain maximum of the cartilage.

The maximum stress is located in the prosthesis. However, at the interface bone-prosthesis local stress peaks and stress free zones occur. The magnitude of these local stress peaks is similar to those in the case of the normal pelvis, so only the stress free zones are of special interest due to the possible occurrence of bone remodification.

The analysis shows a decreasing stress magnitude within the bone with an increasing Young's modulus of the prosthesis material. For high modulus material (metal prostheses), a larger reduction of stress in the bone is calculated (Figure 6).

It has to be considered that trabecular bone and cortical bone behave differently. The stresses in the trabecular bone show a larger degradation than the stresses in the cortical bone. This difference increases

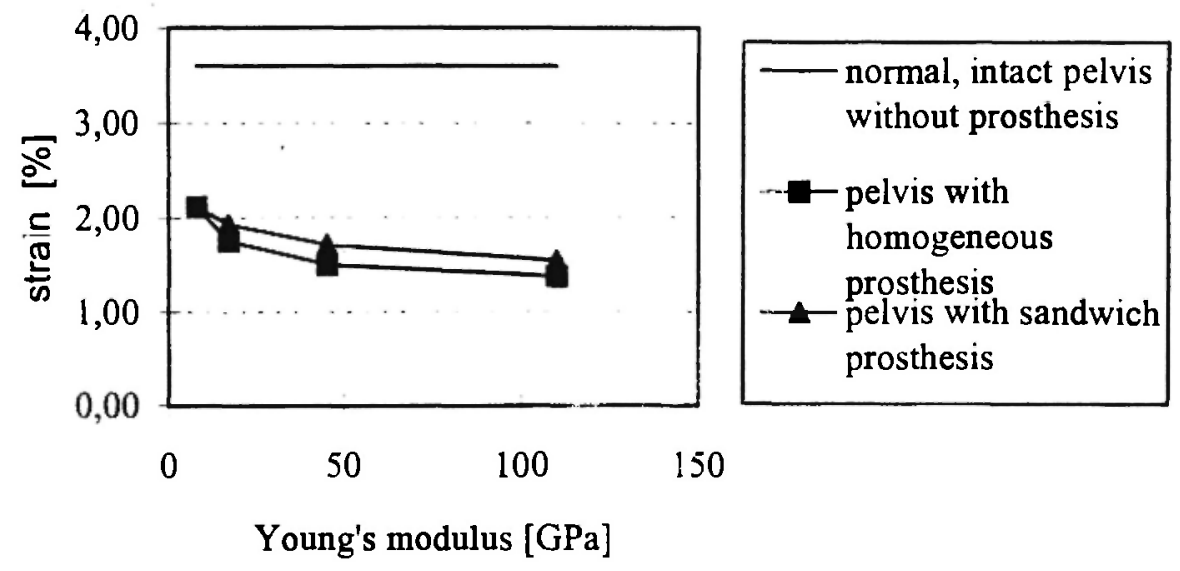

Fig. 5: Stain in the pubic symphysis dependent on the stiffness of the prosthesis material.

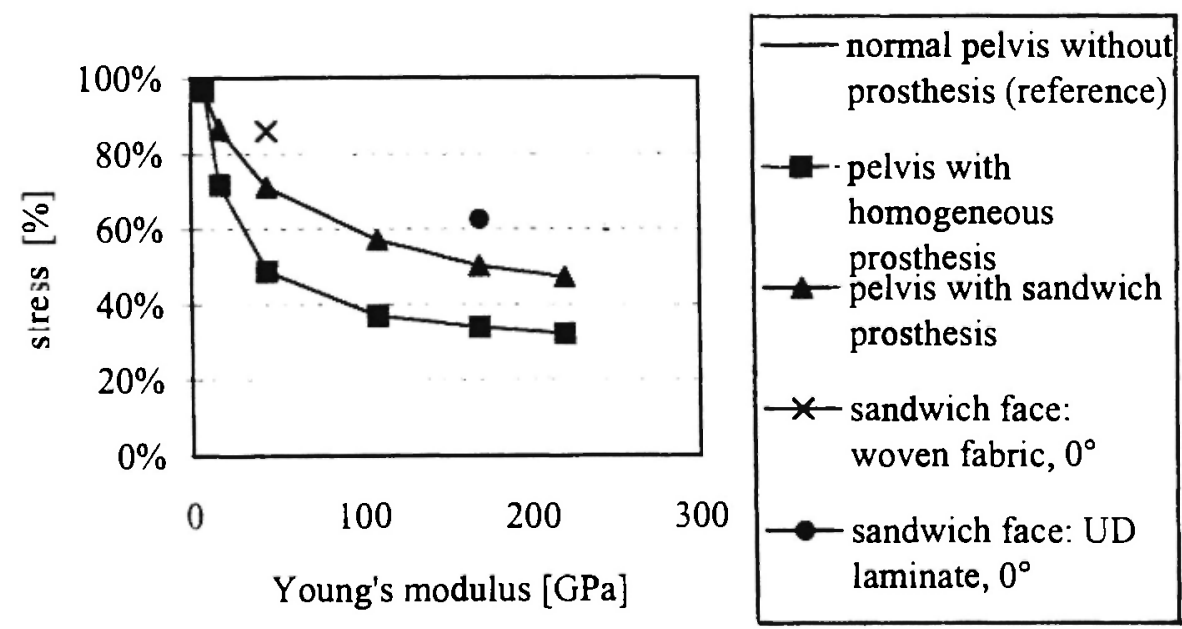

Fig. 6: Stress of the bone dependent on the stiffness of the prosthesis material. 
for prostheses with a higher Young's modulus. It can be interpreted as a shift in the load transfer. With increasing Young's modulus, the load is transferred concentrated through the cortical bone.

\section{DISCUSSION}

The deformation of the pelvis, in particular the ilium wings and the ischium (Figure 4), shows similarities to the deformation of the hemi-pelvic model of Hansen $/ 6 /$. The motion of the symphysis is a complex motion in three-dimensional space with large strains in the cartilage. This large deformation of the pubic symphysis is also simulated by Ortloff et al. $7 /$ although they used one value for both trabecular and cortical bone. This mean value of the Young's modulus for trabecular and cortical bone results in a similar global deformation behaviour but in a different stress distribution in the bone.

The stress distribution concerning trabecular and cortical bone, however, is important for the joining method between the prosthesis and the bone. Here a qualitative agreement is found with analyses of Oonishi $18 \%$.

The deformation behaviour of the pelvis with a homogeneous prosthesis shows that a material with a Young's modulus equal to the mean modulus of different bone types (6 GPa) should be used.

The simulation of the pelvis with a sandwich prosthesis, including anisotropic material properties for the prosthesis' outer surface, shows that the occurring stress distribution in the bone at the interface is closer to the stress situation of the intact pelvis (Figure 6). Here a carbon fibre reinforced medical polymer offers a good opportunity as prosthesis material due to its excellent biocompatibility, X-ray transparency and the adaptive mechanical properties.

With this study, just a part of the design process is investigated. The finite element method is a suitable tool to give qualitative recommendations for the design of a custom made composite prosthesis.

The next steps are the full automation of the mesh generation and the integration of this part in the design process.

In addition some fundamental research is needed to obtain quantitative insight into the stress distribution in the bone depending on the Young's modulus of the prosthesis.

\section{ACKNOWLEDGEMENTS}

The authors wish to thank Dr.-Ing., Dipl.Wirtsch.-Ing. W. Siebels of MAN Ceramics Ltd. in Deggendorf, Germany, for his assistance and support.

\section{REFERENCES}

1. M.R.S. Huisman. Mechanical analysis for the design of a fiber reinforced pelvic prosthesis, Diploma Thesis, Eindhoven University of Technology, The Netherlands and Institut fur Verbundwerkstoffe $\mathrm{GmbH}$, Kaiserslautern, Germany.

2. A. Seireg and R.J. Arvikar. The prediction of muscular load sharing and joint forces in the lower extremities during walking, J. Biomechanics, 8, 89-102 (1975).

3. T.M. Keaveny and W.C. Hayes. A 20-year perspective on the mechanical properties of trabecular bone, J. Biomech. Eng., 115, 534-542 (1993).

4. M. Dalstra, R. Huiskes, A. Odgaard and L. Erning. Mechanical and textural properties of pelvic trabecular bone, J. Biomechanics, 26, 523-535 (1993).

5. MAN Ceramics Ltd., Deggendorf, Germany: Material Data Brochure.

6. T.M. Hansen and J.B. Koeneman. Biomechanical 3D analysis of the human pelvis, in: 4th International ANSYS Conference and Exhibition, Part I, 15.60-15.78, 1989.

7. H. Ortloff, H.M. Tensi, H. Gese and P. Stütz. Deformation of the human pelvis during walking by means of 3D-FEM - Consequences for pelvic reconstruction with implants. Submitted to $J$. Biomechanics, 1994.

8. S. Oonishi, H. Isha and T. Hasegawa. Mechanical analysis of the human pelvis and its application to the artificial hip joint - by means of the three dimensional finite element method, J. Biomechanics, 16, 427-444 (1983). 
